\title{
Prevalence of Mineral Bone Disease in Dialysis Patients.
}

\author{
Dr. Nabiha Rizvi, Faiza Tasleem, Ulfat Altaf. \\ Department of Nephrology \\ Hijaz Hospital, Lahore, Pakistan
}

\begin{abstract}
:
Objective

The objective of the study was to find out the prevalence of bone mineral disease in dialysis patients.

Background

Progressive loss of kidney function in chronic kidney disease (CKD) leads to reduced production of 1- $\alpha-(\mathrm{OH}) 2-\mathrm{D} 3$ (1,25-dihydroxyvitamin D; calcitriol) and abnormal mineral homeostasis leading to bone mineral disorders. The disorder starts with stage 2 CKD but manifests itself as the disease progresses until stage 3 CKD.

Material and methods

This study was carried out on 61 patients with end-stage renal disease on regular HD at our Centre. After obtaining informed consent, complete blood count, blood urea level, serum creatinine level, albumin level, uric acid level, Ca level, phosphate level, alkaline phosphatase level, and PTH level were done.

Results

Out of 60 patients $41(68.33 \%)$ were high bone turnover, $10(16.6 \%)$ were normal bone turnover whereas $9(15 \%)$ were low bone turnover.

Conclusion

The prevalence of CKD-MBD among dialysis patients is $83.33 \%$ at our center and needs proper monitoring by routine investigations including serum phosphate, calcium, vitamin D levels and most importantly i-PTH levels according to guidelines.
\end{abstract}

Key Words: Chronic kidney disease, CKD, mineral bone disease, hyperparathyroidism, hemodialysis, parathyroid hormone, calcium, phosphorus.

\section{Correspondence to:}

Dr. Nabiha Rizvi

Department of Nephrology

Hijaz Hospital, Lahore, Pakistan

Email: rizvinabiha@gmail.com

PJKD 2020;(11):214-218

\section{Introduction}

The prevalence of chronic kidney disease (CKD) is estimated at $8 \%$ to $16 \%$ of the total worlds' population. $\mathrm{CKD}$ is associated with numerous morbidities and complications, one of which is mineral and bone disorder (CKD-MBD). ${ }^{1}$ CKD- MBD is a complex systemic disease observed in patients with estimated glomerular filtration rates (eGFRs) of $60 \mathrm{~mL} / \mathrm{min}$ or lower. Alterations in the control mechanisms for calcium and phosphorus homeostasis occur early in the course of CKD and progress as kidney function decreases. ${ }^{2}$

The disorder has three components:

1. Biochemical abnormalities in concentrations of calcium, phosphorus, parathyroid hormone (PTH), and 1,25- dihydroxyvitamin D3 [1,25(OH)2D3].

2. Abnormalities in bone, including turnover, mineralization, and volume, and or decreased linear growth and strength.

3. Extra skeletal calcification. ${ }^{3}$

Pathophysiology

In normally functioning kidneys, about $90 \%$ of plasma phosphate is filtered and excreted. However, in patients with CKD, disorders that lead to CKD-MBD (including secondary hyperparathyroidism, hyperphosphatemia, decreased intestinal calcium absorption and 
disordered vitamin D metabolism). It begins in stage 2 CKD but does not become clinically detectable until late stage 3 or early stage $4 .^{4-6}$

Levels of i-PTH are regulated by a negative feedback loop with serum calcium levels. Low serum calcium stimulates the parathyroid glands to secrete i-PTH; high serum calcium inhibits PTH secretion. However, in patients with CKD, low calcium levels stimulate parathyroid gland to secrete PTH and osteocytes and osteoblasts secrete increased levels of fibroblast growth factor 23 (FGF23). ${ }^{7}$ FGF23 maintains normal serum phosphate levels by reducing phosphate reabsorption in the tubules. FGF23 also reduces phosphate absorption in the small intestine through decreased vitamin D (calcitriol) production. ${ }^{8}$ These mechanisms maintain normal serum calcium and phosphorus levels early in CKD, even as the patient's PTH level rises. The patient's serum calcium level generally remains within normal limits until GFR declines to less than 50 $\mathrm{mL} / \mathrm{min} / 1.73 \mathrm{~m} 2 .{ }^{8,9}$ These changes result into four types of histopathological patterns of bone disease as recognized in CKD 5:

Hyper parathyroid bone disease: High bone turnover disease that is attributed to untreated secondary hyperparathyroidism (SHPT). It is represented by bone anomalies such as cortical bone thinning and increased abnormal trabecular bone.

Adynamic bone disease: Absent or low bone resorption and formation and could be an early finding of CKD. It is often associated with low PTH level and the patients are more vulnerable to develop fractures.

Low turnover of bone with an increased unmineralized osteoid matrix that in turn will lead to decrease bone strength. It is often attributed to deficiency of vitamin D, metabolic acidosis and hypocalcemia.

Mixed renal osteodystrophy Combined mineralization defects and high bone turnover defects. $^{10}$

These alterations in bone turnover contribute to decreased skeletal mechanical competence, which can result in fractures and diminished capacity to bear loads. The most common fractures are fragility fractures of the vertebrae, femoral neck of the hip, or the wrist (Colles fractures). ${ }^{11}$ The other consequence of CKD-BMD is cardiovascular disease which is the leading cause of death in patients with CKD. Hyperphosphatemia has been shown to stimulate osteoblasts and vascular calcification, particularly of arteries and cardiac valves. ${ }^{1}$

\section{Monitoring Recommendation}

KDOQI recommends beginning to monitor the metabolic abnormalities that accompany CKDMBD in stage 3. To assess bone health without doing a biopsy, KDIGO recommends measuring serum PTH or bone-specific alkaline phosphatase. ${ }^{12}$ Markedly high or low values of these parameters indicate the degree of bone turnover. In addition to evaluating bone with PTH level, clinicians must screen patients for biochemical abnormalities associated with secondary hyperparathyroidism, including hypocalcemia, hyperphosphatemia, and calcidiol level. ${ }^{12}$

The current study was conducted to assess the level and type of BMD at our maintenance hemodialysis unit.

\section{Patients and Methods}

This study was carried out on 60 patients with end-stage renal disease (ESRD) on regular HD in our dialysis center at Hijaz hospital. Informed consent was taken from all included patients. This study was done during the period from September to November 2019. The study excludes 
patients having acute infections, connective tissue disease, malignancy, thyroid gland dysfunctions, recent myocardial infarction, recent trauma in preceding 6 months.

All studied patients were subjected to full history and laboratory investigations including serum albumin, total Ca level, serum phosphate level, serum alkaline phosphatase level, and serum iPTH level. The diagnosis of MBD among the studied patients was made according to the following: suggestive MBD-related symptoms (bone pain, muscle weakness, and combination of both symptoms), and pathological fractures), and MBD-related laboratory indicators (serum PTH, serum Ca, serum phosphorus, and serum alkaline phosphatase levels).

The studied patients were divided into three groups according to serum PTH level:

1. Low bone turnover: PTH level below $150 \mathrm{pg} / \mathrm{ml}$.

2. Normal bone turnover:PTH level between 150 and $300 \mathrm{pg} / \mathrm{ml}$.

3. High bone turnover: PTH level more than $300 \mathrm{pg} / \mathrm{ml}$.

\section{Results}

The study revealed $50(83.3 \%)$ patients with CKD-MBD. Out of total 60 patients $41(68.33 \%)$ were high bone turnover and $10(16.6 \%)$ were normal bone turnover whereas $9(15 \%)$ were low bone turnover as shown in table1

Table 1: Mineral Bone Disease types in 60 maintenance hemodialysis patients.

\begin{tabular}{|l|l|l|}
\hline & Number of patients & Percentage \\
\hline Total patients & 60 & $100 \%$ \\
\hline High bone turnover & 41 & $68.33 \%$ \\
\hline Normal bone turnover & 10 & $16.6 \%$ \\
\hline Low bone turnover & 9 & $15 \%$ \\
\hline
\end{tabular}

The pattern of calcium and phosphate levels in three different patterns of bone disease is expressed in table 2 .

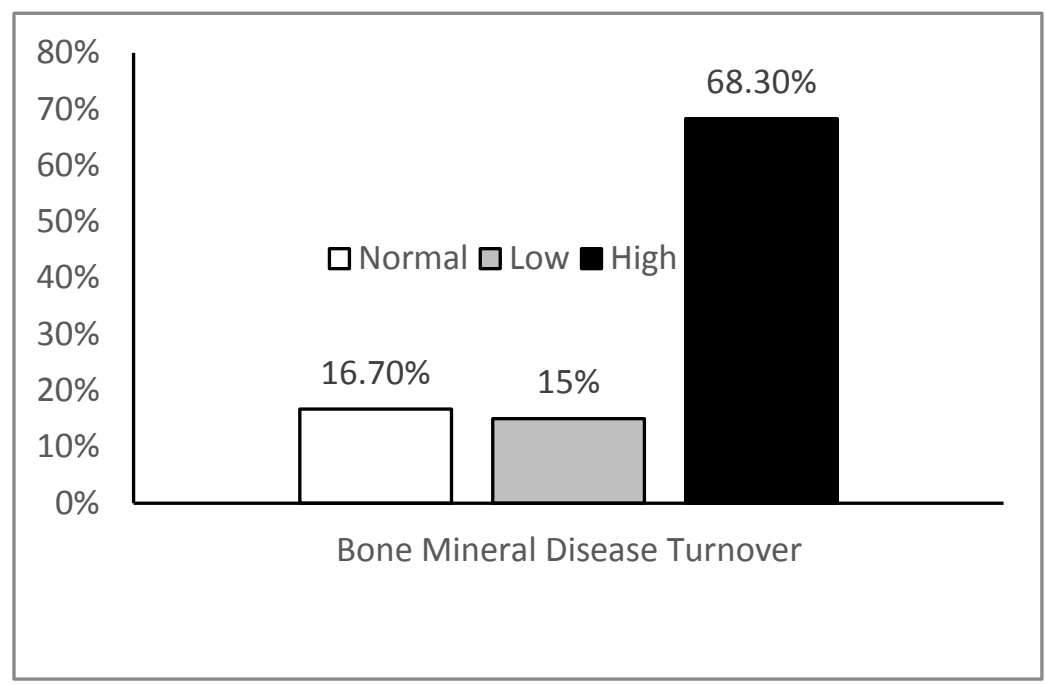

Figure 1: Bar diagram showing the percentage of patients with different types of bone mineral disease

\section{Discussion}

The study revealed majority, 41(68.33\%) patients with high bone turnover and 41 patients there were $29(70.7 \%)$ who had hypocalcemia, 11 (26.8\%) had normal calcium levels whereas only 
one patient (2.4\%) had borderline high calcium level. Phosphate levels in $35(85.3 \%)$ of these 41 patients were high whereas $6(14.7 \%)$ had normal levels. The reason for this can be that therapy with vitamin D was ineffective, and evaluation for the use of other vitamin D analogues and the possible need of surgical intervention of the parathyroid glands for tertiary hyperparathyroidism has to be considered. In the other group with high PTH and low serum Ca levels, the lack of vitamin $\mathrm{D}$ effectiveness could be either undertreatment with vitamin $\mathrm{D}$ or poor patient compliance. $^{13}$

Table 2: serum calcium and phosphorus levels and type of mineral bone disease in 60 hemodialysis patients.

\begin{tabular}{|c|c|c|c|}
\hline & $\begin{array}{l}\text { Low bone } \\
\text { turnover }\end{array}$ & $\begin{array}{l}\text { High bone } \\
\text { turnover }\end{array}$ & $\begin{array}{l}\text { Normal bone } \\
\text { turnover }\end{array}$ \\
\hline \multirow{3}{*}{$\begin{array}{ll}\text { Ca } & <8.5 \\
& 8.5-9.5 \\
& >9.5\end{array}$} & 1 & 29 & 4 \\
\hline & 6 & 11 & 6 \\
\hline & 1 & 1 & \\
\hline \multirow{3}{*}{$\begin{array}{cc}\text { PO4 } & <2.5 \\
& 2.5-4.5 \\
& >4.5 \\
\end{array}$} & & & 1 \\
\hline & 5 & 6 & 2 \\
\hline & 4 & 35 & 1 \\
\hline
\end{tabular}

The other group of low bone turnover had 6 patients $(66.6 \%)$ had borderline high,1 $(16.6 \%)$ had high and $(16.6 \%)$ had low serum calcium levels whereas phosphate in 4 patients $(44.4 \%)$ was high $n=5(66.6 \%)$ had normal levels. In this study, patients with low bone turnover disease had higher mean serum Ca level compared with the mean serum Ca levels in both the hyper parathyroid bone disease group and the patients with no laboratory evidence of bone disease. Most of these patients are using calcium supplements and high doses of vitamin D analogues without prescription (personal experience).

The nature and type of renal bone disease vary from one patient to another, and is multifactorial and the gold standard for diagnosis of renal bone disease is bone biopsy but its invasive nature as well as its cost and overall complexity has withdrawn it from the clinical practice. ${ }^{14}$ Hence, serum markers of bone turnover have been used to evaluate bone turnover in patients with CKD. ${ }^{15}$ Consequently, serum PTH level has taken the leading position among the noninvasive tools for assessing renal bone disease. ${ }^{16}$

\section{Conclusion}

The prevalence of CKD-BMD among dialysis patients was high in our study, majority being high turnover. Proper monitoring and management of BMD will lead to avoidance of complications associated with it.

\section{Conflict of interest: None declared}

\section{References}

1. Moschella C. Guidelines for diagnosis, treatment, and management. J A Acad PA. 2016;29(7):21-29.

2. Martin KJ and Esther A. Metabolic Bone Disease in Chronic Kidney Disease. González. JASN 2007, 18 (3) $875-885$.

3. Moe S, Drueke T, Cunningham J, Goodman W, Martin K, Olgaard K, et al. Definition, evaluation, and classification of renal osteodystrophy: A position statement from Kidney Disease: Improving Global Outcomes (KDIGO). Kidney Int 69 : $1945-1953,2006$

4. Fang Y, Ginsberg C, Seifert M, Agapova O, Sugatani T, Register TC et al. CKD-induced wingless/ integration 1 inhibitors and phosphorus cause the CKD-mineral and bone disorder. J Am Soc Nephrol. 2014;25(8):1760-1773. 
5. Uhlig K, Berns JS, Kestenbaum B, Kumar R, Leonard MB, Martin KJ et al. KDOQI US commentary on the 2009 KDIGO clinical practice guideline for the diagnosis, evaluation, and treatment of CKD-mineral and bone disorder (CKD-MBD). Am J Kidney Dis. 2010;55(5):773-799.

6. Martin KJ, González EA. Metabolic bone disease in chronic kidney disease. J Am Soc Nephrol. 2007;18(3):875-885.

7. Isakova T, Wolf MS. FGF23 or PTH: which comes first in CKD. Kidney Int. 2010;78(10):947-949.

8. Oliveira RB, Cancela AL, Graciolli FG, Dos Reis LM, Draibe SA, Cuppari L et al. Early control of PTH and FGF23 in normophosphatemic CKD patients: a new target in CKD-MBD therapy. Clin J Am Soc Nephrol. 2010;5(2):286-291.

9. Hasegawa H, Nagano N, Urakawa I, Yamazaki Y, Iijima K, Fujita T, et al. Direct evidence for a causative role of FGF23 in the abnormal renal phosphate handling and vitamin D metabolism in rats with early-stage chronic kidney disease. Kidney Int. 2010;78(10):975-980.

10. Osama Mosbah. Chronic Kidney Disease-Mineral and Bone Disorders (CKD-MBD). Archives of Nephrology and Urology 2 (2019): 033-051.

11. American Academy of Orthopaedic Surgeons. recommendations for enhancing the care of patients with fragility fractures. www.aaos.org/CustomTemplates/Content. Accessed November 30, 2019.

12. KDIGO clinical practice guideline for the diagnosis, evaluation, prevention, and treatment of chronic kidney disease-mineral and bone disorder (CKD-MBD). Kidney Int Suppl. 2009;(113):S1-S140.

13. Buargub MA, Nabulsi MF, Shafeh TA. Prevalence and pattern of renal osteodystrophy in chronic hemodialysis patients: a cross sectional study of 103 patients. Saudi J Kidney Dis Transplant 2006; 17:401407.

14. Eknoyan G, Levin A, Levin NW. Bone metabolism and disease in chronic kidney disease. Am J Kidney Dis 2003; 42(Suppl): 1-201.

15. Martin K, Olgaard K. Diagnosis, assessment and treatment of bone turnover abnormalities in renal osteodystrophy. Am J Kidney Dis 2004; 43:558-565.

16. Barreto FC, Barreto DV, Moyses RM, Neves KR, Canziani ME, Draibe SA, et al. K/DOQI-recommended intact PTH levels do not prevent low-turnover bone disease in hemodialysis patients. Kidney Int 2008; 73:771-777. 